\title{
Detection of temporal order of noise-like luminance functions
}

\author{
HERMAN P. SNIPPE and JAN J. KOENDERINK \\ Utrecht University, Utrecht, The Netherlands
}

\begin{abstract}
We study the capacities of human observers to time order light sources that emit dynamic noise, identical for the different light sources, except for an adjustable delay. There is a range of temporal delays for which human observers are perfectly able to perform this task, using the direction of the motion percept that is evoked by the stimulus as a cue. An optimal delay between light sources at which the observers are most robust against any deterioration of the stimulus is defined. We claim that optimal delays (15-25 msec) correspond to the time delay of a putative Reichardt correlation mechanism in human motion vision. Contrary to the ability of human observers to sense temporal correlations in noise sequences, observers are totally unable to detect anticorrelation between noise sequences. This inability rules out motion opponency as a viable model for human front-end ("early") motion vision.
\end{abstract}

Human motion perception is often studied with the use of a discrete temporal presentation of optical patterns. Especially well studied is the case of two-frame apparent motion, in which a spatial luminance pattern is presented once and after some time (the delay) is presented again in a spatially shifted position. The spatial structure of the optical pattern can be continuous (e.g., a sine-wave grating) or semicontinuous (e.g., Julesz-patterns, or random pixel arrays), or it can consist of dense patterns of randomly placed dots (random dot patterns). The minimum and maximum displacements (step sizes) under which apparent motion is perceived have been determined as a function of a large number of parameters: delay (see, e.g., Baker \& Braddick, 1985b), stimulus size (e.g., Baker \& Braddick, 1982; De Bruyn \& Orban, 1989; Nakayama \& Silverman, 1984), spatial frequency content (e.g., Chang \& Julesz, 1983; De Bruyn \& Orban, 1989; Nakayama \& Silverman, 1984), eccentricity of presentation (e.g., Baker \& Braddick, 1985a), and so forth. Yet in contrast with apparent motion, which has received much interest, the inverted situation in which a (continuous or semicontinuous) temporal pattern is presented at a few number of spatial discrete positions (points) has been much neglected. Experiments with this space-time inverted apparent motion paradigm have been done only with very simple temporal luminance functions: a flash (van Santen \& Sperling, 1984; Westheimer, 1983; Westheimer \&

\footnotetext{
This research was supported by the InSight project of the ESPRIT Basic Research Actions of the European Community, and by the Dutch Foundation for the Advancement of Pure Research (NWO). J.K.K. is affiliated with the Utrecht Biophysics Research Institute at the Buys Ballot Laboratory of Utrecht University. Correspondence should be addressed to H. P. Snippe, Department of Psychology, University of Stirling, Stirling FK9 4LA, U.K.
}

-Accepted by previous editor, Charles W. Eriksen
McKee, 1977), a pulse of some extended duration (McKee \& Taylor, 1984), and a harmonic (sine-wave) function (van Santen \& Sperling, 1984). For all these elementary functions, there exists a minimum delay for the temporal presentations at the different spatial points, for which perceived simultaneity gives way to a perceived temporal order. There is no use in trying to measure a maximum delay at which a correct perception of temporal order is lost again. For the harmonic functions, aliasing effects do occur, but these are due entirely to the repetitive nature of the stimulus and are thus not informative about the observer's visual system; and for the pulsed stimuli, the correct perception of temporal order continues indefinitely with increasing delay. ${ }^{1}$

In the experiments reported here, we used dynamic noise for all our temporal luminance functions. That is, spatially discrete (point-like) light sources radiated temporal noise-like luminance functions that were correlated perfectly between the light sources, except for an adjustable delay. If the delay becomes very small, the observer is still able to sense the correlations, but perceives them as simultaneous; he/she is not able to order the relative timing any more. On the other hand, when the delay becomes large, the observer is not able to perceive the correlations at all, so the ability to order the light sources with respect to time is also lost. Hence there is a finite range of delays for which the observer is able to perceive the spatiotemporal structure of the stimulus. Moreover, this psychophysically determined range of delays corresponds perfectly to the introspective sensation of visual motion. That is, if the observer does not perceive motion, he/she is unable to judge the relative temporal delay, and if the observer does perceive motion, the direction of perceived motion always corresponds with the sign of the temporal delay. Therefore, we believe that although formally we are studying the perception of temporal order in spatially distinct stimuli, this is also a study in the 
spatiotemporal limits of human front-end ("early") motion perception.

Front-end motion vision is often modeled in terms of a temporal correlation operation (Adelson \& Bergen, 1985; Reichardt, 1961; van Santen \& Sperling, 1985). Our finding that under suitable conditions human observers are able to perform such a correlation provides empirical support for these schemes. Moreover, the delay range within which observers are able to sense motion direction in our stimuli provides information about the temporal parameters of these putative mechanisms.

An often discussed issue in the study of human motion vision is motion opponency. Are observers able to independently access motion detectors tuned to opposite motion directions, or is only the difference of outputs of motion detectors tuned to opposite directions available (van Santen \& Sperling, 1985)? In Appendix C, we show that for a spatial configuration of two light sources, such a motion opponent scheme would predict a reversal of perceived motion if we reversed the contrast polarity of the dynamic luminance variations of one of these light sources. However, in Experiment 2, we find that human observers do not conform to this prediction, thus ruling out motion opponency as a model for human front-end motion vision.

\section{Method}

\section{Stimuli}

The stimulus consisted of a row of $N(2, \ldots 6)$ red light-emitting diodes (LEDs) horizontally spaced (equidistant at $1 \mathrm{~cm}$ ) on a pin board. To generate a noise-like signal, we employed a maximum length 32-bit shift register (Golomb, 1967) that provides a sequence of essentially uncorrelated random bits (see Figure 1). These sequences are also known as $m$ sequences. See Sutter (1987) for an application in electrophysiology. The shift register is sampled at a number $N(2, \ldots 6)$ of equidistant bits that provide the temporal signal for the $N$ LEDs on the pin board. Because different LEDs are driven by different bits in the shift register, the temporal luminance signals emitted by the LEDs are delayed with respect to each other. The delay $T$ between consecutive LEDs is determined by the spacing $n$ in bits in the shift register that drive them, and by the adjustable clock frequency $f$ of the shift register (the rate at which the bits shift in the shift register):

$$
T=n / f \text {. }
$$

On a given trial, the shift-register-driven luminance variations start and stop simultaneously for the different LEDs in the display. Thus a mathematical description of the spatiotemporal structure of our stimuli is

$$
L(x, t)=W(t)\left[\sum_{m=1}^{N} P(x-m s) M(t-m T)\right],
$$

with $P$, the point-like spatial structure of the individual light sources (LEDs); $s$, the spatial separation of consecutive LEDs; $M$, the noiselike temporal structure of the luminance radiated by the light sources; $T$, the temporal delay between consecutive LEDs of these dynamic luminance functions; and, finally, $W(t)$, the temporal presentation window (rectangular, with duration $d$ ). Because $W$ is equal for the different LEDs, delay information can only be extracted from the relative timing of the dynamic shift-register-driven luminance func-

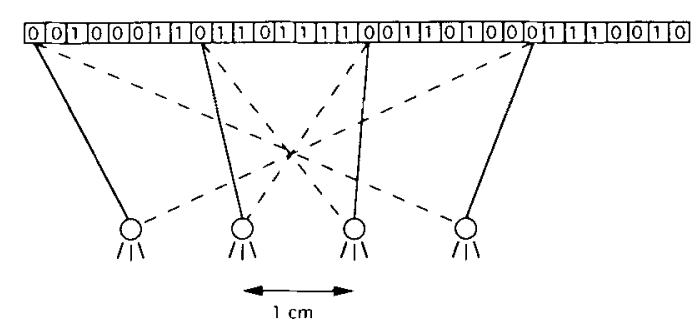

Figure 1. A scheme of the stimulus set up. In a shift register, a sequence of bits ( 0 or 1$)$ steps one place to the right $f$ times per second. The new value of the left most bit is determined from a logical operation (modulo 2 addition) on the values of a number of selected bits. A suitable selection rule for the bits that enter the modulo 2 addition makes the shift register maximum length: it repeats itself only after $2^{32}-1$ shift operations.

The shift register is sampled at a number $N$ of places with equidistant spacing $n$ ( $n=8$ in the figure) that drive $N$ equidistant LEDs visible to the observer $(N=4$ in the figure). A bit value 0 at the driving place means that the LED is off at the moment; $a$ bit value 1 means that the LED is on. Because the signal shifts rigidly in the shift register, the temporal luminance functions for the different LEDs are identical, except for a delay $T=n / f$ between consecutive LEDs. The LED time response is easily fast enough to follow the highest clock frequencies that we used.

If a single LED is driven by the shift register, all the observers introspectively see is a light source (the LED) emitting a noise-like dynamic luminance signal (of course).

If two or more LEDs are driven by the shift register, however, the observers have a clear sensation of motion flow between the LEDs (at suitable values for the delay $T$, and for a large enough stimulus presentation time, contrast, etc.).

An independent random generator determines the sign of the delay at a given trial by driving an electronic switch that determines whether the solid or dashed connections between the shift register and the LEDs are used. In practice, the observer uses the direction of perceived motion for a response: rightward for the solid connections, leftward for the dashed connections.

tions $M$ for the different LEDs, and not from the timing of the window $W$ in which there is any dynamic luminance at all. This is important, because it is known that observers are very sensitive to asynchronies in such luminance envelope functions (McKee \& Taylor, 1984)

\section{Psychophysical Procedure}

To determine the minimum and maximum delays $\left(T^{-}\right.$and $T^{+}$, respectively) for which the observer is still able to time order the stimulus, we employed the method of constant stimuli. At a fixed delay $T$, a run of 30 presentations occurred, each presentation having a random sign for the delay. The sign at a trial was determined by the random generator of an Atari 1040ST microcomputer. The task for the observer was to determine the sign of the delay. In practice, as noted in the introduction, this was done on the basis of the direction (leftward or rightward) of perceived motion. The observer entered his answer on the computer keyboard, after which the computer initiated (with a fixed 500 -msec pause) the next presentation. After each 30-trial run was finished, the computer evaluated the percentage of correct answers given by the observer. Delay values $T$ for the different runs were spaced one octave apart, and were obtained by varying the clock frequency of the clock that drove the shift register (Equation 1). Figure 2 gives an example of the psychometric functions obtained. Threshold delays $\left(T^{-}\right.$and $\left.T^{+}\right)$were defined at $\mathbf{8 0 \%}$ correct answers and were determined by linear in- 
terpolation from the percentage correct answers at delay values that resulted in percentage correct answers in the range $65 \%-90 \%$.

\section{Stimulus Parameters and Viewing Conditions}

To get a feeling for the ability of human observers to judge temporal order with the novel stimulus that we employ, we determined threshold delays $T^{-}$and $T^{+}$over a broad range of conditions. We varied the following parameters:

Number (N) of LEDs: $N=2,3,4$, or 6 . This should give some indication of the dependence of observer capacities on the amount of spatial information in the stimulus. In particular, would observers be able to perform the task when only the bare minimum of spatial information necessary to define motion direction ( $N=2$ LEDs) was present?

Presentation time d: $d=100,200,400$, or $1,000 \mathrm{msec}$. This enabled us to study the temporal integration of motion information in human vision for our spatially extremely sparse stimuli.

Sampling distance $\mathrm{n}: n=1,2,4,8$. As detailed in Appendix A, $n$ is related to the (internal) contrast of the dynamic luminance variations.

Separation of consecutive LEDs in the stimulus. Physical separation was always $1 \mathrm{~cm}$. We used two foveal viewing distances: $1 \mathrm{~m}$ (inter-LED separation $34^{\prime}$ ) in which the discrete spatial structure is easily perceived, and $14 \mathrm{~m}$ for which the inter-LED separation $\left(2.5^{\prime}\right)$ was more comparable to the human foveal spatial acuity. At the small $(1-\mathrm{m})$ viewing distance, we also used an eccentric $\left(22.5^{\circ}\right.$ horizontal eccentricity) condition. Acuity at $22.5^{\circ}$ is about $16^{\prime}$ (Weymouth, 1958).

Stimulus blur. Spatial blur of the stimulus was either absent or present. To generate stimulus blur, we used a piece of ground glass placed in front of the LEDs that generated a radially symmetric spatial luminance distribution with a standard deviation $\sigma \approx 0.3 \mathrm{~cm}$ for each of the LEDs in the display.

The design was factorial; that is, we studied every combination of the parameters listed above (resulting in a total of $4 \times 4 \times 4$ $\times 3 \times 2=384$ conditions). In addition, Observer H.S. also performed the foveal, large-separation (34') no-blur condition without his correcting spectacles, thus introducing $-4 \mathrm{D}$ of spectacle blur.

\section{Observers}

Two observers were used throughout the experiment. Observer H.S. (one of the authors), male, 32 years, is experienced in psychophysical experiments concerning motion vision. Observer A.S., female, 25 years, underwent extensive practice before the start of the formal data collection, and was knowledgeable about the purposes of the experiment. Both observers used their normal optical correcting devices, except in the case noted above for observer H.S. Viewing was always binocular.

\section{RESULTS}

\section{Experiment 1}

\section{Correlated Dynamic Noise}

As described in the Method section, psychometric curves like those in Figure 2 enable us to extract an upper limit $T^{+}$and a lower limit $T^{-}$for the range of delay values for which the observer is able to perform the task. These limits turn out to be well-defined functions of the parameters used in this experiment: the number $N$ of light sources, the presentation time $d$, and the sampling distance $\boldsymbol{n}$ in the shift register (related to stimulus contrast as it is discussed in Appendix A). Some examples of these relations are presented in Figure 3.

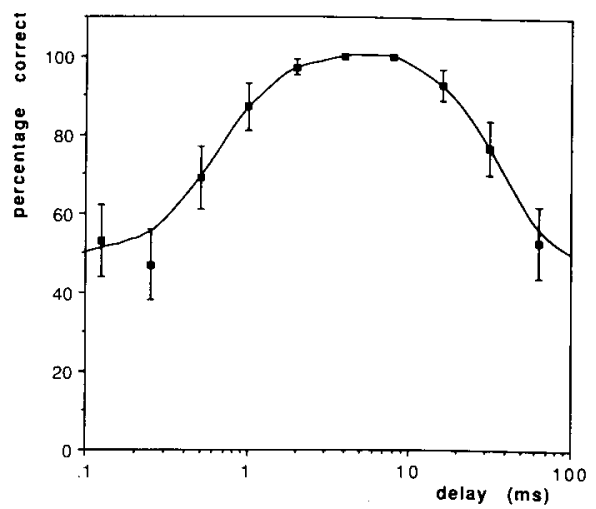

Figure 2. An example of the psychometric curves collected in Experiment 1. The variability bars are the standard deviations due to the binomial nature of the response distribution: $\sigma=$ $N_{t}^{-1 / 2}[p(1-p)]^{1 / 2}$, with $p$ the fraction correct responses and $N_{t}$ the number of trials at a certain delay $\left(N_{t}=30\right.$ throughout the experiments).

Note that the dependence of observer performance on the experimental parameters agrees with prior intuition: the delay range for which the task can be performed increases if the presentation time $d$ increases, if the number $N$ of light sources increases, or if the contrast increases (i.e., with decreasing $n$ ). Also note that the improvement takes place at both the lower $\left(T^{-}\right)$and the upper ends of the delay range $\left(T^{+}\right)$. Finally, note that a number of data points in Figure 3 are missing. In general, this is a consequence of the stimulus conditions' being too adverse for the observer to reach criterion ( $80 \%$ correct) performance at any delay value $T$. This explains the absence of data points at the shortest presentation time $d=$ $100 \mathrm{msec}$ for the lowest contrast $n=8$ in Figure 3A, for $N=2$ LEDs and $n \geq 4$ in Figure 3B, and for $N=2$ and 200 -msec presentation time and 100 -msec presentation time at any $N$ in Figure $3 \mathrm{C}$. The absence of data for the upper delay limit $T^{+}$for the case in which consecutive LEDs were driven by consecutive bits in the shift register (i.e., $n=1$ ) in Figures $3 \mathrm{~A}$ and $3 \mathrm{~B}$ has another reason, however. In this condition, Observer H.S. was often able to maintain percentage correct responses around threshold criterion (80\%) even with very long delays (at least a couple of hundreds of milliseconds), thus making the determination of $T^{+}$problematical. Although this observer lost a strong percept of smooth motion at these long delays, he could still time order the LEDs with some confidence on the basis of a much more jerky motion percept. We encountered this problem of the combination of a loss of a smooth motion percept and a psychophysical function that refused to go to $50 \%$ (chance) only for $\mathrm{Ob}$ server H.S. in the $n=1$ condition (LEDs driven by consecutive bits in the shift register). For all $n>1$ for Observer H.S. and for any $n$ for Observer A.S., if there was a delay range with percentage correct responses larger 

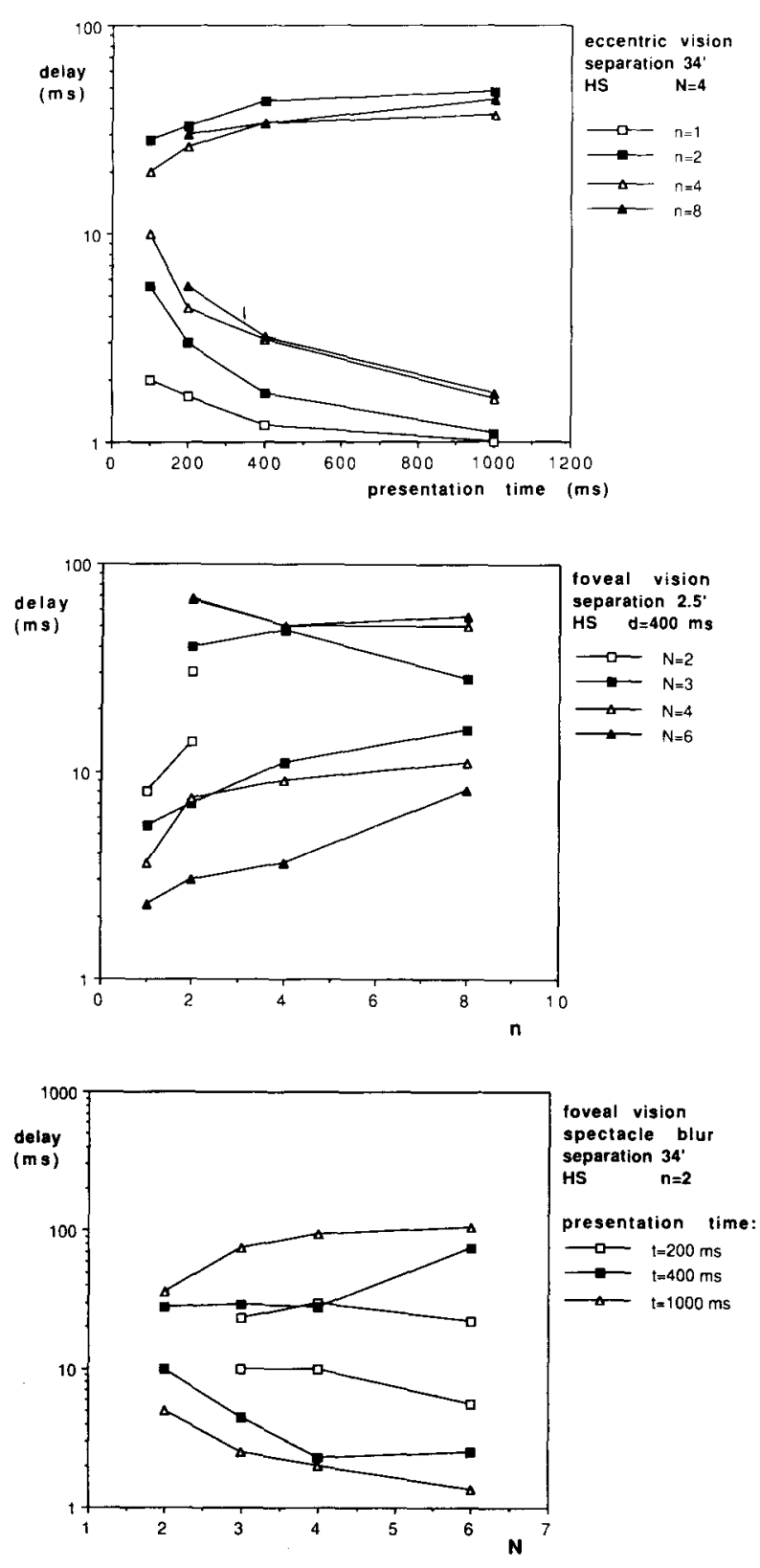

Figure 3, Some examples of the dependence of the upper and lower delays on the experimental parameters.

than $80 \%$, we could always unambiguously determine a delay value $T^{+}$beyond which any motion percept was lost, and percentage correct responses dropped rapidly to $50 \%$ (chance level). We excluded data for $T^{+}$for Observer H.S. in the $n=1$ condition in all analyses in this paper.

Because of the large amount of data gathered, it is impossible to present them all in the format of Figure 3, so some data reduction is required. To do this, we will describe our data set in terms of a power law behavior for the dependence of delay thresholds $T^{ \pm}$on the experimental parameters $N, n$, and $d$ :

$$
\begin{aligned}
T^{-} & =T_{0}^{-}(N-1)^{-\alpha_{-}}(n)^{\beta_{-}}(d)^{-\gamma_{-}} \\
T^{+} & =T_{0}^{+}(N-1)^{\alpha}+(n)^{-\beta_{+}}(d)^{\gamma_{+}}
\end{aligned}
$$

(units of presentation time $d$ are seconds in these equations). Note that we choose the signs in the power expressions so that the exponents should be positive, and that we use the number of nearest neighbor pairs $N-1$ instead of the number $N$ of light sources itself. We want to stress that we do not attach any great theoretical importance to the structure of Equations 3 and 4 . We present them merely as a useful means of data compression. As such, however, they are very efficient. This can be seen as follows. The raw threshold delays $T^{-}$vary over a 20fold range, and the $T^{+}$values vary over a 5 -fold range. In comparison with these huge variations, the variations in the values for $T_{0}^{-}$and $T_{0}^{+}$obtained using the estimates for the power exponents $\alpha, \beta$, and $\gamma$ that we shall derive later in this section are very small. We find that the resulting distributions of $T_{0}^{ \pm}$at a fixed value of the remaining stimulus parameters (viewing distance and eccentricity, and presence or absence of stimulus blur) have a coefficient of variation (ratio of standard deviation and mean of the distribution) of $29 \%$ for Observer A.S. and $24 \%$ for Observer H.S. (averaged over these remaining stimulus parameters). Moreover, we argue in Appendix B that the stochastic nature of the binomial distribution of elementary left/right answers given by the observer should already give rise to a variation of about $20 \%$ in the empirically determined threshold delays. Any other probabalistic source of noise would only increase this figure, so any residual systematic trends in $T_{0}^{ \pm}$would be very hard to determine. Therefore, we conclude that it makes little sense to try to refine the data description used in Equations 3 and 4 within the parameter ranges explored here, unless one is willing to spend a much larger amount of observation time to reduce the stochastic errors in the experimental determination of the threshold delay values $T^{-}$and $T^{+}$.

We determined the best-fitting exponents in Equations 3 and 4 by using a linear least squares fitting procedure. The results are presented in Table 1 . Because they turned out to be not statistically different (at a $5 \%$ confidence level) for the 2 observers, we averaged their values and used the average as presented in Table 2 for our further analysis.

With the exponents from Table 2 and the empirically determined threshold delays $T^{-}$and $T^{+}$, we used Equations 3 and 4 to calculate the values of the quantities $T_{0}^{-}$ and $T_{0}^{+}$, averaged over $N, n$, and $d$, for all viewing conditions (viewing distance and eccentricity with stimulus blur either present or absent). We present the results in Table 3. Some comments are in order. First, the maximum delay threshold $T_{0}^{*}$ is remarkably stable across conditions. For both observers, $T_{0}^{+}$is typically about $50 \mathrm{msec}$. There is more variation in the lower delay threshold $T_{0}^{-}$. For the eccentric condition and the foveal condition with small (2.5') LED separation, $T_{0}^{-}$does not critically depend on stimulus blur. $T_{0}^{-}$is about $5 \mathrm{msec}$ for the $2.5^{\prime}$ separation foveal condition and about $1.5 \mathrm{msec}$ for the 
Table 1

The Power Exponents (With Standard Errors) That Describe the Dependence of the Delay Limits on the Stimulus Parameters $N$, $n$, and $d$

\begin{tabular}{ccccccc}
\hline Subject & $\alpha_{-}$ & $\alpha_{+}$ & $\beta_{-}$ & $\beta_{+}$ & $\gamma_{-}$ & $\gamma_{+}$ \\
\hline A.S. & $0.62 \pm 0.15$ & $0.24 \pm 0.23$ & $0.55 \pm 0.12$ & $0.21 \pm 0.09$ & $0.51 \pm 0.07$ & $0.28 \pm 0.12$ \\
H.S. & $0.70 \pm 0.11$ & $0.39 \pm 0.06$ & $0.39 \pm 0.10$ & $0.19 \pm 0.06$ & $0.71 \pm 0.08$ & $0.42 \pm 0.06$ \\
\hline
\end{tabular}

eccentric condition. For the foveal condition with the large (34') LED separation, however, there is a huge improvement (decrease) of the $T_{0}^{-}$value when spatial stimulus blur is introduced. We have reason to suspect that this is not an effect of stimulus blur per se, however. Results on asynchronicity detection with conventional (flash-like) temporal luminance functions show that foveal detection thresholds deteriorate for spatial separations larger than about $10^{\prime}$ (Westheimer \& McKee, 1977), consistent with the elevated values for $T_{0}^{-}$we find in the unblurred $34^{\prime}$ separation foveal condition. When we introduce a spatial blurring of the stimulus, we create physical dynamic luminance correlations at spatial separations smaller than the nominal 34' LED separation. Presumably the decrease of asynchronicity detection thresholds upon the introduction of stimulus blur arises because the observer now uses the correlations at these smaller separations that are more favorable according to the results of Westheimer and McKee (1977).

\section{Experiment 2}

\section{Anticorrelated Dynamic Noise}

We performed a second experiment with our dynamic noise stimuli to assess whether observers would be able to determine temporal order in noise sequences that were anticorrelated between LEDs. For this reason, we provided one LED with a noise sequence and a second LED (again at a horizontal separation of $1 \mathrm{~cm}$ ) with a delayed and inverted copy of this noise sequence (that is, we interchanged 1 and 0 in the noise sequence). The observers were unable to perform the task under any condition. Every combination of viewing condition, contrast, presentation time, and delay value that we used resulted in chance performance. Also, all the observers saw introspectively were two, seemingly uncorrelated noise sequences. As we argue in detail in Appendix C, this inability of observers to sense motion in anticorrelated noise sequences is strong evidence against a motion detection scheme in which observers are able to access only the difference response of motion detectors tuned to opposite motion directions, and not the individual direction selective detector responses themselves.

Table 2

The Between-Observer Average of the Power Exponents in Table 1

\begin{tabular}{cccccc}
\hline \multicolumn{7}{c}{$\alpha_{-}$} & $\alpha_{+}$ & $\beta_{-}$ & $\beta_{+}$ & $\gamma_{-}$ & $\gamma_{+}$ \\
\hline $\mathbf{0 . 6 6}$ & 0.32 & 0.47 & 0.20 & 0.61 & 0.35 \\
\hline
\end{tabular}

\section{A REICHARDT CORRELATION SCHEME}

The fact that observers are able to perceive motion in our space sampled dynamic noise stimuli has a natural explanation in terms of a Reichardt model for motion perception (Figure 4). A Reichardt detector senses the temporal correlation of signals sampled at two different locations (Reichardt, 1961; van Santen \& Sperling, 1984), exactly the aspect of motion that survives in our spatially discrete, but temporally effective continuous stimuli.

Assuming a Reichardt (correlation) principle for the detection of motion direction (and hence temporal order) in our stimuli, one should realize that already in an elementary Reichardt detector (Figure 4) there exist a number of time constants. First, there is the time constant $\sigma_{t}$ in the temporal prefiltering operation TF, which is both biologically inevitable, but also advantageous in filtering out unwanted, aliasing temporal components in the input signal (van Santen \& Sperling, 1985). Next, there is the delay parameter $\tau$ for one of the two input lines for the correlator. ${ }^{2}$ Finally, one also expects a temporal integration (low-pass) operation $\int$ after the correlation to suppress the time-dependent part of the correlator output and so convert it into a constant (time-independent) motion signal (Nakayama, 1985a).

We believe that the results from Experiment 1 yield information on each of these parameters in a correlation model. We will consider these parameters one by one.

\section{The Delay Parameter $\tau$}

The Reichardt correlator in Figure 4 responds optimally if the physical delay $T$ in our experiment equals its internal delay $\tau$ (for only then are both of the input signals to the correlator in Figure 4 fully correlated). Thus, we expected optimal performance if $T=\tau$ in our experiment. Because we only determined minimum and maximum threshold delays in our experiment (e.g., $T_{0}^{-}$and $T_{0}^{+}$in Table 3), it would seem that all we could say about $\tau$ is that it should be somewhere in this range: $T_{0}^{-}<\tau<$ $T_{0}^{+}$. However, using the following argument, we can make a more precise estimate of $\tau$ from the results in Experiment 1 . Suppose we deteriorate the stimulus conditions (i.e., reduce contrast, presentation time, and/or number of light sources) to increase $T^{-}$and decrease $T^{+}$until the delay range for which the observer can perform the time ordering task (at $80 \%$ correct) shrinks down to a single delay point $T$ (i.e., we have $T^{-}=T^{+}$in this condition). This is the physical delay value for which the observer is most robust against the stimulus deterioration. There- 


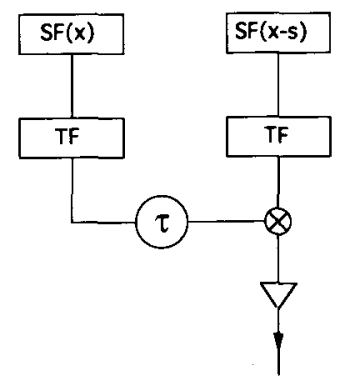

Figure 4. A plausible implementation of an elementary Reichardt detector. The dynamic luminance distribution is sampled by two spatial filters (receptive fields) $\mathrm{SF}(x)$ and $\mathrm{SF}(\mathrm{x}-\mathrm{s})$, which are shifted copies of each other with spatial shift $s$, the detector span. Next, both signals pass through temporal filters TF and feed into the correlator $\otimes$, one signal directly, the other after a delay $\tau$. The basic idea of Reichardt correlation is that if the input consists of an optical pattern $I\left(x-v_{0} t\right)$ rigidly moving at the detector's tuning speed $v_{0}=$ $s / \tau$, both input signals to the correlator are identical, and hence, maximally correlated. The operation $\nabla$ performed on the correlator output is a lowpass temporal filter (integrator) that filters out phase-dependent variations in the correlator output. For our spatially sampled stimuli, we expect the detector to respond maximally if its span $s$ equals the separation between consecutive LEDs in our experiment $\left(2.5^{\prime}\right.$, respectively $\left.34^{\prime}\right)$, and if the physical delay $T$ between consecutive LEDs equals the detector delay parameter $\tau$.

fore, this delay value would be a reasonable estimate for $\tau$. In general, the value of $\tau$ so determined would depend on which stimulus parameter (i.e., $N, n$, or $d$ ) one chooses to deteriorate, and at what values for the other parameters. However, a close inspection of Table 1 shows that, owing to a remarkable coincidence, this is not the case for our data set. The reason for this is that the ratio of the + and - exponents in Equations 3 and 4 is about equal for $\alpha, \beta$, and $\gamma$. We show the ratios in Table 4 . The mean ratio is $2.07 \pm 0.14$. For ease of exposition, we use a value of 2 for the ratio in the remainder of our presentation. Now, equating $T^{+}=T^{-}=\tau$ in Equations 3 and 4 to determine the optimal delay $\tau$ at which $T^{-}$and $T^{+}$coincide under deterioration of the stimulus conditions, one finds, by multiplying Equation 3 with the square of Equation 4 (due to the "fact" that $\alpha_{-} / \alpha_{+}=\beta_{-} / \beta_{+}=\gamma_{-} / \gamma_{+} \approx 2$ ):

$$
\tau^{3}=\left(T_{0}^{-}\right)\left(T_{0}^{+}\right)^{2}
$$

Thus,

$$
\tau=\left(T_{0}^{-}\right)^{1 / 3}\left(T_{0}^{+}\right)^{2 / 3} .
$$

In Table 5, we present the values for $\tau$ derived in this fashion. There is a significant dependence of $\tau$ on the viewing condition used, with $\tau$ clearly lowest for an eccentric $\left(22.5^{\circ}\right)$ presentation. For the foveal condition with the small LED separation $\left(2.5^{\prime}\right)$ and for the eccentric condition, there is no significant change in the value of $\tau$ upon spatial blurring of the stimulus. In the foveal condition with large LED separation (34'), however, there is an obvious reduction of the critical delay $\tau$ when the stimulus is spatially blurred. There is little between-subject difference in the critical delay values. Of six values to be compared, only one (the foveal unblurred condition with small LED separation) shows a statistically just significant difference; the other five values show no significant differences between observers.

The delay values $\tau$ that we report in Table 5 are in excellent agreement with optimal delay values (18-24 msec) reported by Burt and Sperling (1981) in a multipath competetive apparent motion design, and also with the optimal delay value of $17 \mathrm{msec}$ reported by Werkhoven and Koenderink (1991) in a two-alternative competetive apparent rotary motion design. Baker and Braddick (1985b), in a two-frame apparent motion experiment with random dot patterns report an optimal delay value $(40 \mathrm{msec})$ larger than our results. However, their optimal delay values were mainly derived at upper values for the displacements of the spatial pattern for which the observer could still consistently report motion direction (jump sizes about $\left.40^{\prime}-50^{\prime}\right)$. Given our result that for foveal vision the values for the optimal delay are increased (to $\tau \approx 30 \mathrm{msec}$ ) at the largest LED separation that we used $\left(34^{\prime}\right)$, we think that there is no conflict. Our delay values $\tau$ are somewhat smaller than the temporal gating values $(20-70 \mathrm{msec})$ that give rise to a severe loss of motion perception in the time-

Table 3

Minimum and Maximum Delay Threshold Parameters $T_{0}^{-}$and $T_{0}^{+}$(in Milliseconds)

\begin{tabular}{|c|c|c|c|c|c|c|c|}
\hline \multirow[b]{4}{*}{ Subject } & \multirow[b]{4}{*}{ Stimuli } & \multicolumn{6}{|c|}{ LED Separation } \\
\hline & & \multicolumn{4}{|c|}{ Foveal } & \multirow{2}{*}{\multicolumn{2}{|c|}{$\frac{\text { Eccentric } 22.5^{\circ}}{34^{\prime}}$}} \\
\hline & & \multicolumn{2}{|c|}{$2.5^{\prime}$} & \multicolumn{2}{|c|}{$34^{\prime}$} & & \\
\hline & & $T_{0}^{-}$ & $T_{0}^{+}$ & $T_{0}^{-}$ & $T_{0}^{+}$ & $T_{0}^{-}$ & $T_{0}^{+}$ \\
\hline $\begin{array}{l}\text { A.S. } \\
\text { H.S. }\end{array}$ & $\begin{array}{l}\text { no blur } \\
\text { ground glass blur } \\
\text { no blur } \\
\text { ground glass blur } \\
\text { spectacle blur }\end{array}$ & $\begin{array}{l}4.2 \pm 0.3 \\
5.8 \pm 0.8 \\
4.5 \pm 0.2 \\
5.4 \pm 0.6\end{array}$ & $\begin{array}{l}48 \pm 2 \\
46 \pm 4 \\
56 \pm 2 \\
54 \pm 4\end{array}$ & $\begin{array}{r}7.3 \pm 0.8 \\
2.2 \pm 0.2 \\
11.3 \pm 1.2 \\
3.9 \pm 0.4 \\
3.5 \pm 0.3\end{array}$ & $\begin{array}{l}55 \pm 4 \\
59 \pm 3 \\
46 \pm 3 \\
50 \pm 3 \\
41 \pm 2\end{array}$ & $\begin{array}{l}1.7 \pm 0.2 \\
1.8 \pm 0.2 \\
1.5 \pm 0.2 \\
1.2 \pm 0.2\end{array}$ & $\begin{array}{l}47 \pm 3 \\
40 \pm 2 \\
45 \pm 2 \\
46 \pm 2\end{array}$ \\
\hline
\end{tabular}
Complete With Their Standard Errors for the Viewing Conditions Used

Note-The delay parameters reported here are representative for the threshold delays at the maximum presentation time ( $d=1,000 \mathrm{msec})$ and contrast $(n=1)$ studied, but with the minimum number $(N=2)$ of LEDs present. Representative threshold delays for other values of the stimulus parameters can be calculated from this table by using Equations 3 and 4 and the power exponents from Table 2 . 
Table 4

The Ratio of the Power Exponents for the Lower and Upper Delay Thresholds

\begin{tabular}{lccc}
\hline \multicolumn{1}{c}{ Subject } & $\alpha_{-} / \alpha_{+}$ & $\beta_{-} / \beta_{+}$ & $\gamma_{-} / \gamma_{+}$ \\
\hline A.S. & 2.6 & 2.6 & 1.8 \\
H.S. & 1.8 & 2.1 & 1.7 \\
$M$, Table 2 & 2.1 & 2.4 & 1.7 \\
\hline
\end{tabular}

gated back and forth motion design used by van Doorn and Koenderink (1982; also Koenderink, van Doorn, \& van de Grind, 1985). The reason van Doorn and Koenderink (1982) and Koenderink et al. (1985) find gating times larger than ours may be that their most detrimental time values are determined not only by $\tau$, but also by the time constant in the integration operation in Figure 4. If the gating window is larger than $\tau$, there is correlation for the detector to detect, but the time during which correlation is present may be too small to allow the buildup of a meaningful velocity signal in the integrator $\int$. Therefore, the most detrimental gating times in the reports of van Doorn and colleagues might be regarded as upper bounds for the delay $\tau$ in a Reichardt model.

\section{Temporal Prefiltering}

The fact that (even for $N=2$ LEDs) we find a range of delay values $\left(T_{-}, \ldots T_{*}\right)$ for which the observer can sense motion in our stimuli can be understood as a consequence of the temporal prefiltering operation (TF in Figure 4). This filter broadens the temporal autocorrelation function of our stimuli (essentially a $\delta$ function for the strings of uncorrelated bits that we generate; see Appen$\operatorname{dix} \mathrm{A}$ ) into a finite temporal range of correlation (Glünder, 1990). As we noted in the introduction, the lower delay limits are in general not a consequence of the loss of a correlation percept: observers are still perfectly able to sense the between-LED correlation, but they perceive them as simultaneous, i.e., they are unable to order them in time. This, presumably, would be a consequence of a statistically equal stimulation of motion detectors tuned to opposite directions. For a Reichardt detector to be stimulated at all for a zero physical delay of the signals delivered, the input filters TF should be slow enough to have an autocorrelation function that is still appreciably different from zero at a time equal to the physiological delay value $\tau$. For a bandfilter TF (e.g., a filter with a bipolar impulse response function), this would mean that the filter TF is slower than predicted from the well-known quadrature relation (delay equal to one fourth of the period of the temporal sine wave optimally transmitted by TF) (Adelson \& Bergen, 1985; van Santen \& Sperling, 1985; Watson \& Ahumada, 1985). A similar deviation from the quadrature relation has been observed for the spatial filtering in motion vision (Boulton, 1990).

On the other hand, the optimal delay values $\tau$ that we report comply very well with the temporal flicker frequencies to which human observers are most sensitive when these two quantities are connected with a quadrature rule. For instance, McKee and Taylor (1984), using spatial stimuli (small line segments) comparable to our point-like LEDs, found optimal frequencies of $12 \mathrm{~Hz}$ in the fovea and $18 \mathrm{~Hz}$ at $20^{\circ}$ eccentricity (as estimated from their Figure 5). Using a quadrature rule, these optimal frequencies translate into delay values of $21 \mathrm{msec}$ in the fovea and $14 \mathrm{msec}$ in the periphery that are in excellent agreement with our estimates of $\tau$ in Table 5. Nakayama (1985a) also drew attention to this agreement of temporal sensitivity functions and delay estimates that occurs when they are connected with a quadrature rule.

A way out of this apparent conflict could be provided through the substitution of a motion energy model (Emerson, Bergen, \& Adelson, 1992) for the Reichardt model in Figure 4. Such a motion energy model has a self-energy term that gives a positive contribution to the detector response for a zero physical delay between LEDs in our stimulus, even if the elementary subfields of the motion detector are in quadrature relation.

Contrary to the lower delay limits, the upper delay limits that we determined in Experiment 1 are a consequence of the loss of a correlation percept. We routinely measured upper delay limits $T^{+}$up to four times the optimal delay values reported in Table 5 . Whether such large delay values can be bridged solely by the broadening of the correlation peak by the temporal prefiltering operation (TF) in either a standard Reichardt or a standard motion energy model remains to be seen. A viable alternative to explain the capacities of our observers at these large (relative to $\tau$ ) delays would be a motion system that consisted of an ensemble of detectors with a range of delay values $\left(\tau_{-}, \ldots \tau_{+}\right)$at a fixed separation (De Bruyn \& Orban, 1988; van de Grind, Koenderink, \& van Doorn, 1992). In this case, the optimal delay $\tau$ that we would measure would

Table 5

Estimates of the Delay Parameter $\tau$ in a Reichardt Correlation Model From the Results in Experiment 1 (in Milliseconds), Together with Standard Errors

\begin{tabular}{llccc}
\hline & & \multicolumn{3}{c}{ LED Separation } \\
\cline { 3 - 5 } Subject & \multicolumn{2}{c}{ Soveal } & Eccentric $\left(22.5^{\circ}\right)$ \\
\cline { 3 - 4 } & \multicolumn{1}{c}{ Stimulus } & $2.5^{\prime}$ & $34^{\prime}$ & $34^{\prime}$ \\
\hline A.S. & no blur & $21.3 \pm 1.0$ & $28 \pm 2$ & $15.5 \pm 1.0$ \\
& ground glass blur & $23 \pm 2$ & $19.9 \pm 0.8$ & $14.4 \pm 0.8$ \\
H.S. & no blur & $24.1+0.6$ & $29 \pm 2$ & $14.4 \pm 0.4$ \\
& ground glass blur & $25 \pm 2$ & $21.2 \pm 1.2$ & $13.6 \pm 0.5$ \\
& spectacle blur & & $18.1 \pm 0.9$ & \\
\hline
\end{tabular}


presumably be related to the delay value represented most densely within such a local pool of velocity detectors. However, the exact relation between the pool range and the optimum delays that we would measure remains to be assessed.

The observers never had a reversed motion percept at the upper end of the delay range: percentage correct answers gradually and monotonically declined from $100 \%$ to $50 \%$ (chance level) without a delay range at which the percentage dropped consistently to a value under $50 \%$ ("temporal aliasing"). Within a Reichardt correlation model, such a reversed motion percept would presumably be a consequence of a negative lobe in the autocorrelation function of the filter TF. The fact that our observers did not perceive reversed motion at any delay could mean that the TF response function has an all-positive autocorrelation function, presumably because TF itself would be monophasic ("sustained"). However, for the motion system, a biphasic ("transient") filter is generally assumed. In this case, the absence of a reversed motion percept throughout the range of physical delays $T$ would presumably mean that the system ignored or discarded any negative output of a Reichardt detector.

\section{Postcorrelation Temporal Integration}

To perform the task in Experiment 1, observers need a presentation time much longer than our estimate $\tau$ for the Reichardt delay to see any motion at all. This can be judged from Figure 3C, for example, where Observer H.S. is unable to perform the time-ordering task with a presentation time of $100 \mathrm{msec}$ (and if only $N=2$ LEDs are present, also for a presentation time of $200 \mathrm{msec}$ ), in comparison with our estimate $\tau=18 \mathrm{msec}$ in Table 5 for this condition. This difference may be caused by the temporal integration operation $\int$ in Figure 4. Remember that the integrator exists to filter out any spurious timedependent output of the correlating element. Applying the quadrature rule mentioned above, one expects the temporal variations of the correlator output signal to be especially pronounced at a repetition rate around $2 \tau$ (not $4 \tau$, because of the quadratic, frequency-doubling operation of the correlator). Assuming that one needs a number of cycles at this repetition rate to get a velocity signal of some reliability, the large presentation times that we find necessary seem quite consistent with a small delay value in a Reichardt correlation model. Also, this result is in full agreement with the slow velocity integration stage hypothesized by Nakayama (1985a) to explain his results on the low cutoff frequency for movement detection of random dot patterns in sinusoidal motion (Nakayama, 1985b).

However, the long presentation times needed in our experiments seem to have been in conflict with reports in the literature that much smaller presentation times are already sufficient for more involved computations on the spatiotemporal luminance pattern (e.g., the metrical judgment of velocity instead of only the sign of the velocity as in our experiment). For instance, De Bruyn and Orban (1988) have reported presentation times of 50-100 msec as enough for subjects to reach (near) asymptotic levels in the estimation of the velocity of a moving random pixel array ("Julesz pattern"), and presentation times as low as $10 \mathrm{msec}$ to be enough for subjects to extract the direction of motion of the pattern. It should be realized, though, that their stimuli were spatially very extensive (typically $10^{\circ}$ in diameter). In this case, the temporal integration in Figure 4 could be exchanged for a spatial integration over detectors. Indeed van Doorn and Koenderink (1983) found evidence of the interchangeability of spatial and temporal integration in motion vision. McKee and Welch (1985) have reported velocity discrimination thresholds for localized stimuli that reach asymptotic levels already at presentation times under $100 \mathrm{msec}$. The spatiotemporal structure of their stimuli (a moving line segment) is very simple compared to that of our noise stimuli, however. For the simple stimuli, the correlation operation in a Reichardt detector reduces to a coincidence detection for which the arguments for the necessity of a temporal integration filter are not valid any more, which could explain the difference in presentation time needed.

\section{DISCUSSION}

\section{Comparison of $T_{0}^{-}$With Previous Results}

In Table 3, we have reported minimum foveal asynchronies of 3-5 msec for the time ordering of dynamic noise-like luminance signals. We can compare this result with a number of reports in the literature.

1. Asynchronicity detection for spatially localized stimuli with a conventional flash-like temporal presentation. Westheimer and McKee (1977) reported thresholds of 3-5 msec; Westheimer (1983) found thresholds of about $5 \mathrm{msec}$. This shows that, given enough contrast and presentation time, observers can time order our novel noiselike dynamic luminance sequences as well as these more conventional flash-like stimuli.

2. $T^{-}$values for two-frame apparent motion with more complicated spatial patterns (random dots) (Baker \& Braddick, 1985a). For the determination of the minimum frame delay necessary to determine the direction of the spatial shift $\left(T^{-}\right)$, Baker and Braddick were somewhat limited by the minimum 5-msec frame duration of their setup. From their Figure 6, however, it can be estimated that the minimum delay for subjects to attain $80 \%$ correct responses is certainly smaller than $10 \mathrm{msec}(5-\mathrm{msec}$ interstimulus intervals +5 -msec frame duration) for a suitable (5'- to $\left.10^{\prime}-\right)$ spatial jump. Using a random dot pattern in a setup in which the individual dots in the display were not synchroneously displaced, Morgan and Ward (1980) reported $T^{-}$values of 2-4 msec for motion direction identification, again in good correspondence with the minimum delay values we have obtained in the present study.

3. Motion direction discrimination for sine-wave stimuli moving behind narrow spatial apertures. Anderson and Burr $(1987,1991)$ have reported that observers can determine motion direction when only 0.03 cycle of a sine wave is visible (if the spatial wavelength is not too small; e.g., spatial frequency $\leq 1 \mathrm{c} / \mathrm{deg}$ ). For the $8-\mathrm{Hz}$ tem- 
poral frequency of their moving sine waves, this 0.03 cycle limit corresponds to a delay of approximately $4 \mathrm{msec}$ between the temporal signals from the right and left parts of the aperture. Again, there is an excellent correspondence with the foveal minimum delay thresholds that we have obtained with our temporally much more complicated stimuli.

For peripheral $\left(22.5^{\circ}\right)$ vision, we report minimum delay values of about $1.5 \mathrm{msec}$ that are considerably lower than our foveal values (at $2.5^{\prime}$ separation) of about $5 \mathrm{msec}$ (see Table 3). McKee and Taylor (1984) also reported lower peripheral asynchronicity detection thresholds ( $3 \mathrm{msec}$ at $20^{\circ}$ eccentricity vs. $5 \mathrm{msec}$ in the fovea). These lower values may be a consequence of the relative fastness of eccentric vision. The temporal modulation transfer function peaks at higher frequencies in the periphery (Kelly, 1984; Koenderink, Bouman, \& Bueno de Mesquita, 1978; Tyler, 1985), which is consistent with the lower estimates for $\tau$ in the periphery than in foveal vision that we report in Table 3. Thus we can explain the 3 -fold ratio between our foveal and peripheral minimum delay thresholds as follows. From Table 5, we find a ratio of 1.6 of our estimated time constants in peripheral and foveal (2.5' separation) vision (averaged over observers and stimulus blur condition). Solely from this, one would already expect a 1.6-fold decrease of the peripheral minimum delay thresholds in comparison with foveal minimum delay thresholds (note that the results of McKee and Taylor, 1984, nicely confirm this prediction). However, we believe that two more factors contributed to a further decrease of the peripheral minimum delay thresholds in our experiment (and not in the experiment of McKee \& Taylor, 1984). First, there was an effect of contrast. As explained in Appendix A, a smaller time constant for some part of the visual system corresponds to a higher perceived contrast for our stimuli. Informally, it was quite obvious during the experiment that the perceived contrast of physically equal stimuli was higher in the periphery. We derive in Equation A14 of Appendix A that this effect would lead to a further decrease of $T^{-}$of about $(1.6)^{0.9}$. The second factor was presentation time. One can argue that in Equation 3 it is reasonable to express presentation time not in terms of a fixed external clock, but in units of the estimated time constant $\tau$. But use of the power exponent $\gamma^{-}=0.61$ from Table 2 leads to a still further reduction of the peripheral minimal delay threshold by a factor $1.6^{0.61}$. Thus, altogether, a 1.6fold reduction of the peripheral time constant can be expected to lead to a 3.2 -fold $\left(1.6^{1+0.9+0.61}\right)$ reduction of the peripheral minimum delay thresholds in comparison with the foveal (2.5' separation) minimum delay thresholds, which is in excellent agreement with the results we report in Table 3.

\section{Comparison of $T_{0}^{+}$With Previous Results}

A number of values of the maximal temporal separation of the two frames for motion direction identification in a two-frame apparent motion sequence have been reported in the literature.
Lappin and Bell (1976) found an 80-msec value for a 1-pixel (4') displacement in a $24 \times 24$ random dot array (see their Figure 2).

Baker and Braddick (1985b) reported a value of $150 \mathrm{msec}$ for a 10 ' displacement in a 'sparse random dot array."

Dawson and DiLollo (1990) have reported values ranging from $50 \mathrm{msec}$ for a $10-\mathrm{cd} / \mathrm{m}^{2}$ background to $150 \mathrm{msec}$ for a $0.1-\mathrm{cd} / \mathrm{m}^{2}$ background.

Finally, Morgan and Ward (1980) in their nonsynchronous apparent motion display found values of $60 \mathrm{msec}$ at normal (artificial) light levels, and $100 \mathrm{msec}$ at dim levels.

The $T^{+}$values that we report in Table $3(40-60 \mathrm{msec})$ are slightly lower than the values from the literature. Note, however, that the values in Table 3 are for the spatially most sparse stimuli with only $N=2$ LEDs present. According to Equation 4 and the value $\alpha^{+}=0.32$ from Table 2 , for $N=6$ one expects an approximately 1.7 -fold $\left(5^{0.32}\right)$ increase in $T^{+}$to values of up to $70-100 \mathrm{msec}$ (see, e.g., Figure 3C). Thus, for the spatially most extended stimuli that we used, the correspondence of our $T^{+}$with values obtained from the literature with spatially much larger stimuli is again good.

\section{No Motion Perception for Anticorrelated Sequences}

In Experiment 2, we have reported that human observers were unable to see anticorrelation between two dynamic light sources. In Appendix C, we will argue that this is an important negative result because, under very broad conditions, it shows that human observers have independent access to direction-tuned motion detectors, and not just to the differential output of motion detectors tuned to opposite directions (see Adelson \& Bergen, 1985; van Santen \& Sperling, 1985).

However, the negative result that we obtained in Experiment 2 also puts some constraints on nonopponent versions of these motion processing schemes. For instance, van Santen and Sperling (1985) show that for a correlation scheme in which the two temporal input filters that feed into the correlator form a quadrature pair (i.e., have a $90^{\circ}$ relative phase shift for any harmonic input), the output of such a "nonopponent" detector is equivalent to the opponent variant. Thus, our result in Experiment 2 also rules out these quadrature versions of a "nonopponent"' Reichardt correlation scheme. What we need is a really nonopponent scheme in the sense that the correlation element gives a strong positive response in the preferred motion direction, but little response for motion in the nonpreferred direction. It can be shown that such schemes exist when one uses input temporal filters in the two input lines for the correlator that are time-shifted (delayed) copies of each other (as in Figure 4). A reversal of input contrast in one of the two input lines for the correlator then leads to a reversal for the detector output of the detector tuned to the "preferred direction," so that this output now becomes strongly negative (while the mirror symmetric detector still shows little response). If we 
now assume that the motion system does not interpret the negative output as corroborative evidence for motion in the reverse direction, but instead simply ignores or discards this negative response (e.g., by applying a half-wave rectification operation to the correlator output), we get a situation that complies with our result in Experiment 2: a strong motion percept for two correlated light sources, and no motion percept when the light sources are anticorrelated.

On the other hand, such a half-wave rectification operation on the output of the quadratic element would not work in a motion energy scheme (because here the nonopponent signals are pure squares, and hence nonnegative; a half-wave rectification would thus have no effect). Thus, while the disappearance (instead of a reversal) of perceived motion in our Experiment 2 has a plausible explanation in a nonopponent Reichardt correlation scheme, it will be much harder to explain even in a nonopponent version of the motion energy scheme.

At first sight, the negative result in Experiment 2 appears to contradict previous results on reverse phi, in which a spatial shift and contrast reversal of a spatial pattern produced a sensation of motion in the direction opposite to the shift (Anstis, 1970). Anstis (1986) has noted that this reverse phi effect can be understood as an aliasing effect generated by the low-frequency part of the pattern upon contrast reversal. Thus, it might be expected that the original reports on reverse phi were due to a preponderance of low spatial frequencies in the patterns used (e.g., luminance edges). According to this explanation, the fact that the relevant (temporal) frequency spectrum of our stimulus did not have this preponderance of low frequencies (it is flat at the lower end of the frequency spectrum) would be the reason for the absence of a reverse phi effect upon introduction of anticorrelation in our experiment. However, reverse phi has also been reported for stimuli with a spatial structure (a random pixel array) very similar to the temporal structure of our stimuli (Sato, 1989). Thus, the comparison of the presence of reverse phi in this reference with the absence of the effect in our study indicates a genuine difference between spatial and temporal processing in human motion vision. We have two suggestions regarding what this difference might be:

1. It is generally assumed that human spatial vision consists of a rather large number of parallel channels that span the spatial frequency axis, but that such a system of parallel channels may not exist for human temporal vision. Following the argument of Anstis (1986), the reverse phi effect might be mediated by the low spatial frequency channel(s) of such a system. However, there is no fundamental reason to expect that these low spatial frequency channels tuned to the reverse direction generate a positive signal for the anticorrelated temporal sequences that we employed, thus explaining the absence of the effect in our study.

2. For the patterns commonly used to demonstrate the reverse phi phenomenon, there is a spatial overlap of first and second patterns presented in the two-frame apparent motion sequence; hence, adaptation effects have to be taken into account (Shioiri \& Cavanagh, 1990). On the other hand, in our experiment, there was no spatial overlap of the luminance sequences to be compared, so that, since spatial adaptation may be extremely local MacLeod, Williams, \& Makous, 1992), adaptation may not have played a similar role in our setup.

\section{Outlook}

Spatially sampled, but temporally effectively continuous patterns have been much neglected stimuli in the study of the visual motion system. New directions in which these stimuli can be employed include the following: velocity discrimination; spatiotemporal resolution of the velocity assignment; the detection and discrimination of optic acceleration; spatial prefiltering of the luminance signal after the sampling operation; and temporal prefiltering of the luminance signal to generate ambiguous motion displays - that is, temporally periodic stimuli that contain correlation peaks in both the normal and the reversed direction.

\section{REFERENCES}

Adelson, E. H., BERGEN, J. R. (1985). Spatiotemporal energy models for the perception of motion. Journal of the Optical Society of America A, 2, 284-299.

ANDERSON, S. J., \& BuRR, D. C. (1987). Receptive field size of human motion detection units. Vision Research, 27, 621-635.

ANDERSON, S. J., \& BurR, D. C. (1991). Spatial summation properties of directionally selective mechanisms in human vision. Journal of the Optical Society of America A, 8, 1330-1339.

Anstis, S. M. (1970). Phi movement as a subtraction process. Vision Research, 10, 1411-1430.

ANSTIS, S. M. (1986). Recovering motion information from luminance. Vision Research, 26, 147-160.

Baker, C. L., \& Braddick, O. J. (1982). The basis of area and dot number effects in random dot motion perception. Vision Research, 22, 1253-1260.

BAKER, C. L., \& BRADDICK, O. J. (1985a). Eccentricity-dependent scaling of the limits for short range motion perception. Vision Research, 25, 803-812.

BAKER, C. L. , \& BRADDick, O. J. (1985b). Temporal properties of the short-range process in apparent motion. Perception, 14, 181-192.

BoultoN, J. C. (1990). The optimal displacement for the detection of motion. Vision Research, 30, 1101-1106.

Burt, P., \& SPERling, G. (1981). Time, distance and feature tradeoffs in visual apparent motion. Psychological Review, 88, 171-195.

Chang, J. J., \& Julesz, B. (1983). Displacement limits for spatial frequency filtered random-dot cinematograms in apparent motion. $\mathrm{Vi}$ sion Research, 23, 1379-1385.

Dawson, M., \& Di Lollo, V. (1990). Effects of adapting luminance and stimulus contrast on the temporal and spatial limits of short-range motion. Vision Research, 30, 415-429.

De Bruyn, B., \& Orban, G. A. (1988). Human velocity and direction discrimination measured with random dot patterns. Vision $R e-$ search, 28, 1323-1335.

De BruYn, B., \& Orban, G. A. (1989). Discrimination of opposite directions measured with stroboscopically illuminated random-dot patterns. Journal of the Optical Society of America A, 6, 323-328.

Emerson, R. C., Bergen, J. R., \& Adelson, E. H. (1992). Directionally selective complex cells and the computation of motion energy in cat visual cortex. Vision Research, 32, 203-218.

Foster, D. H., Gravano, S., \& Tomoszek, A. (1989). Acuity for finegrain motion and for two-dot spacing as a function of eccentricity: Differences in specialization of the central and peripheral retina. $\mathrm{Vi}$ sion Research, 29, 1017-1031.

Glünder, H. (1990). Correlative velocity estimation: Visual motion 
analysis, independent of object form, in arrays of velocity tuned bilocal detectors. Journal of the Optical Society of America A, 7, 255-263.

Golomв, S. W. (1967). Shifi register sequences. San Francisco: Holden-Day.

KELLY, D. H. (1984). Retinal inhomogeneity: I. Spatiotemporal contrast sensitivity. Journal of the Optical Society of America A, 1 , 107-113.

Koenderink, J. J., Bouman, M. A., Bueno de Mesquite, A. E., \& SLAPPENDEL, S. (1978). Perimetry of contrast detection thresholds of moving spatial sine wave patterns: II. The far peripheral visual field (eccentricity $0^{\circ}-50^{\circ}$ ). Joumal of the Optical Society of America, 68, 850-854.

Koenderink, J. J., van Doorn, A. J., \& van de Grind, W. A. (1985). Spatial and temporal parameters of motion detection in the peripheral visual field. Journal of the Optical Society of America A, 2, 252-259.

LAPPIN, J. S., \& BELL, H. H. (1976). The detection of coherence in moving random-dot patterns. Vision Research, 16, 161-168.

MacLeod, D. I. A., Williams, D. R., \& Makous, W. (1992). A visual nonlinearity fed by single cones. Vision Research, 32, 347-363.

MCKeE, S. P., \& TAYLOR, D. G. (1984). Discrimination of time: Comparison of foveal and peripheral sensitivity. Journal of the Optical Society of America A, 1, 620-627.

MCKeE, S. P., \& WELCH, L. (1985). Sequential recruitment in the discrimination of velocity. Journal of the Optical Society of America A, 2, 243-251.

Morgan, M. J., \& WARD, R. (1980). Conditions for motion flow in dynamic visual noise. Vision Research, 20, 431-435.

Moulden, B., Kingdom, F., \& Gatley, L. F. (1990). The standard deviation of luminance as a metric for contrast in random-dot images. Perception, 19, 79-101.

NakAYAMA, K. (1985a). Biological image motion processing: A review. Vision Research, 25, 625-660.

NaKaYAma, K. (1985b). Higher order derivatives of the optical velocity vector field: Limitations imposed by biological hardware. In $\mathrm{D}$. Ingle, M. Jeannerod, \& D. Lee (Ed.,) Brain mechanisms and spatial vision (pp. 59-71). The Netherlands: Martinus Nijhoff.

Nakayama, K., \& Silverman, G. H. (1984). Temporal and spatial characteristics of the upper displacement limit for motion in random dots. Vision Research, 24, 293-299.

REICHARDT, W. (1961). Autocorrelation, a principle for the evaluation of sensory information by the central nervous system. In W. A. Rosenblith (Ed.), Sensory communication (pp. 303-317). New York: Wiley.

SATO, T. (1989). Reversed apparent motion with random dot patterns. Vision Research, 29, 1749-1758.

Shiorri, S., \& CavanaGH, P. (1990). ISI produces reverse apparent motion. Vision Research, 30, 757-768.

Stone, L. S., Watson, A. B., \& Mulligan, J. B. (1990). Effect of contrast on the perceived direction of a moving plaid. Vision Research, 30, 1049-1067.

SUTTER, E. (1987). A practical non-stochastic approach to nonlinear time-domain analysis. In Advances in methods of physiological systems modelling (Vol. 1). Los Angeles: University of Southern California Press.

TYLER, C. W. (1985). Analysis of visual modulation sensitivity: II. Peripheral retina and the role of photoreceptor dimensions. Journal of the Optical Society of America A, 3, 393-398.

VAN de Grind, W. A., Koenderink, J. J., \& Van Doorn, A. J. (1992). Viewing distance invariance of movement detection. $E_{x}$ perimental Brain Research, 91, 135-150.

VAN Doorn, A. J., \& KoENDERINK, J. J. (1982). Temporal properties of the visual detectability of moving spatial white noise. Experimental Brain Research, 45, 179-188.

VAN DOORN, A. J., KoENDERINK, J. J. (1983). Spatiotemporal integration in the detectability of motion. Vision Research, 23, 47-56.

VAn Santen, J. P. H., \& SPERling, G. (1984). Temporal covariance model of human motion perception. Journal of the Optical Society of America A, 1, 451-473.

VAN SANTEN, J. P. H., \& SPerling, G. (1985). Elaborated Reichardt detectors. Journal of the Optical Society of America A, 2, 300-321.

W Atson, A. B. (1982). Derivation of the impulse response: Comments on the method of Roufs and Blommaert. Vision Research, 22. 1335-1337.

Watson, A. B., \& Ahumada, A. J. (1985). Model of human visualmotion sensing. Journal of the Optical Society of America A, 2, 322-344.

Werkhoven, P., \& Koenderink, J. J. (1991). Reversed rotary motion perception. Journal of the Optical Society of America A, 8, 1510-1516.

Westheimer, G. (1983). Temporal order detection for foveal and peripheral stimuli. Vision Research, 23, 759-763.

Westheimer, G., \& MCKeE, S. P. (1977). Perception of temporal order in adjacent visual stimuli. Vision Research, 17, 887-892.

Weymouth, F. W. (1958). Visual sensory units and the minimal angle of resolution. American Journal of Ophthalmology, 46, 102-113.

\section{NOTES}

1. For very small separations (smaller than the acuity threshold at the eccentricity at which the stimuli are presented), there does exist an upper delay at which the observer loses the ability to time order these pulse stimuli (Foster, Gravano, \& Tomoszek, 1989). However, because this occurs only for these small separations, it is impossible to measure this upper limit for well-separated stimuli as we have done in the present study.

2. In a biological system, the temporal prefiltering operation TF and the delay $\tau$ will often be combined in a single temporal filter. Conceptually, however, it is perfectly alright to keep them separated.

\section{APPENDIX A Contrast Effects}

In Experiment 1, we determined the dependence of threshold delay values $T \pm$ on the spacing $n$ of the bits in the shift register that drive consecutive LEDs. In Appendix A, we will derive some consequences of variations in this parameter. Specifically, we will argue that $n$ is related to the perceived contrast of our stimuli.

The physical contrast $c$ for our temporal luminance functions $M(t)$ emitted by individual LEDs (Equation 2) was always $100 \%$ :

$$
c^{2}=\frac{\left\langle\left[M(t)-\langle M>]^{2}\right\rangle\right.}{<M\rangle^{2}}=1,
$$

for our pseudorandom $m$-sequences $M(t)$ that consist of strings of uncorrelated bits for which $50 \%$ are "on" $[M(t)=M]$ and $50 \%$ are "off" $[M(t)=0]$. The averages " $<>$ " in Equation A1 are temporal. We use a root mean square (RMS) definition for contrast, which is very reasonable for our noise-like stimuli (Moulden, Kingdom, \& Gatley, 1990).

Note that Equation A1 holds independently of the clock frequency $f$ that determines the presentation duration $b$ of individual bits in the $m$-sequence: $b=1 / f$. This is at variance with the percept of our observers: perceived contrast decreases with decreasing bit duration $b$ (i.e., increasing $f$ ). We can resolve this apparent conflict as follows. Suppose the dynamic luminance $M(t)$ passes through an internal temporal filter TF with pulse response function

$$
h\left(t ; t_{0}\right)=h\left(\frac{t}{t_{0}}\right)
$$

The parameter $t_{0}$ governs the time scale of the internal response function. After passage through TF, the physical dynamic luminance $M(t)$ transforms into an internal luminance $\vec{M}(t)=M(t)$ $* h\left(t / t_{0}\right)$. We denote internal quantities with a tilde in this appendix; " $*$ " is the convolution operation. $\tilde{M}$ has an associated internal contrast 


$$
\tilde{c}=\left(\frac{<[\tilde{M}(t)-<M>]^{2}>}{<\tilde{M}(t)>^{2}}\right)^{1 / 2} .
$$

One can easily prove

$$
\begin{aligned}
<\tilde{M}> & =\frac{1}{2} M \int h\left(t / t_{0}\right) d t \\
& =\frac{1}{2} M t_{0} \int h(y) d y .
\end{aligned}
$$

For the calculation of the numerator in Equation A3, we proceed as follows:

$$
\tilde{M}(t)-<\tilde{M}>=\int M^{\prime}\left(t-t^{\prime}\right) h\left(t^{\prime}\right) d t^{\prime},
$$

where we define $M^{\prime}(t)=M(t)-1 / 2 M$. Thus,

$$
[\tilde{M}(t)-<\tilde{M}>]^{2}=\iint M^{\prime}\left(t-t^{\prime}\right) M^{\prime}\left(t-t^{\prime \prime}\right) h\left(t^{\prime}\right) h\left(t^{\prime \prime}\right) d t^{\prime} d t^{\prime \prime} .
$$

Hence

$$
\begin{gathered}
\left\langle[\tilde{M}(t)-<\tilde{M}>]^{2}>\right. \\
=\frac{1}{2 B} \int_{-B}^{B} \iint M^{\prime}\left(t-t^{\prime}\right) M^{\prime}\left(t-t^{\prime \prime}\right) h\left(t^{\prime}\right) h\left(t^{\prime \prime}\right) d t^{\prime} d t^{\prime \prime} d t \\
=\iint A\left(t^{\prime}-t^{\prime \prime}\right) h\left(t^{\prime}\right) h\left(t^{\prime \prime}\right) d t^{\prime} d t^{\prime \prime},
\end{gathered}
$$

where $A$ is the autocorrelation function of our dynamic luminance. For our strings of uncorrelated bits, $A$ is essentially the autocorrelation function of an individual bit in $\boldsymbol{M}^{\prime}$. Individual bits in $M^{\prime}$ have a height $\pm M / 2$ and a width (duration) $b$. Hence, $A$ is triangular with height $M^{2} / 4$ and total width $2 b$ (Figure A1).

In Figure A2, we plot internal contrast $\tilde{c}$ as a function of bit duration $b$ using Equation $\mathrm{A} 4$ for the mean and Equation $\mathrm{A} 7$ for the variance of the internal signal. For the pulse response function $h(t)$, we choose an expression proposed by Watson (1982):

$$
h(t)=u(t)\left[\left(\frac{t}{A}\right)^{8} e^{-t / A}-\frac{1}{B}\left(\frac{t}{C}\right)^{9} e^{-t / c}\right] .
$$

This response function is bipolar, with the amount of inhibition in the negative tail governed by the parameter $B$. McKee and Taylor (1984) estimated values for the parameters $A, B$, and $C$ from the temporal modulation transfer function by using spatial stimuli (small bars) similar to our point-like stimuli. For

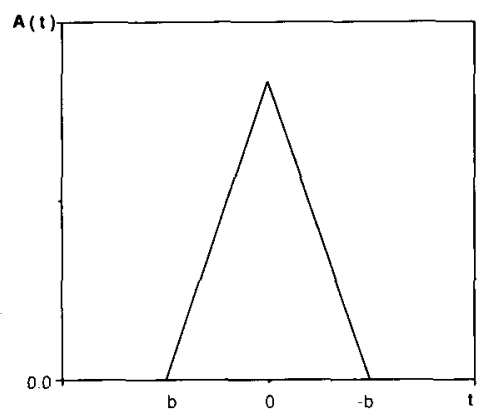

Figure A1. The autocorrelation function $A(t)$ of a string of uncorrelated bits where individual bits have duration $b$.

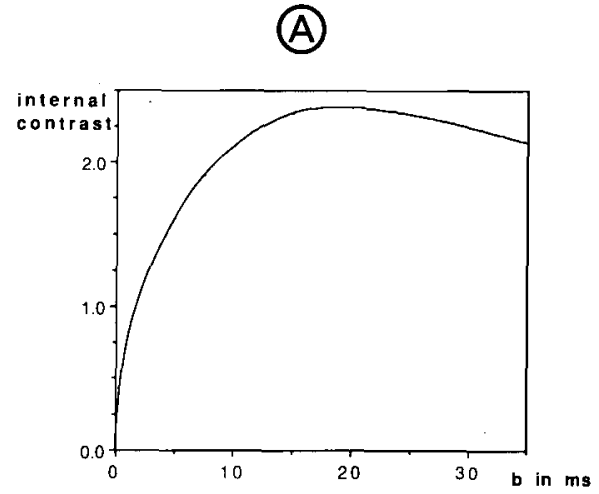

(B)

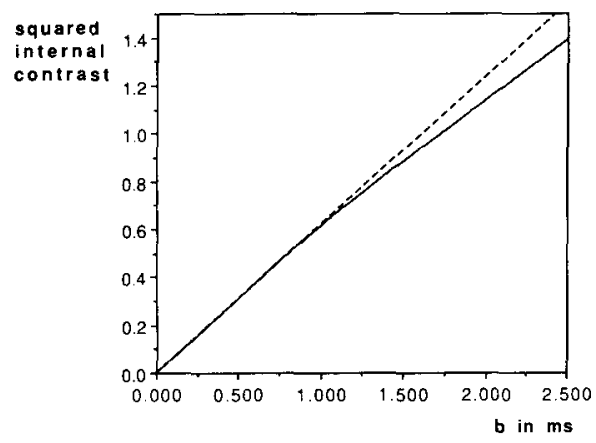

Figure A2. (A) Internal contrast $\tilde{c}$ for our stimuli, determined as described in the text, as a function of duration $b$ of individual bits in the $m$-sequence. (B) For small values of $b$, squared internal contrast $\tilde{c}^{2}$ is linear in $b$.

our Figure A2, we used the estimates from their Table 1 for Observer L.W. in the fovea: $A=3.64 \mathrm{msec}, C=4.26 \mathrm{msec}$, and $B=12$.

We note a number of aspects in Figure A2:

1. For sufficiently small values of $b$, the plot of $\tilde{c}^{2}$ versus $b$ is linear (see Figure A2B). The decrease of $\tilde{c}$ with decreasing $b$ (increasing $f$ ) is in agreement with the introspective reports of our subjects. An alternative understanding of the linearity of the initial part of Figure A2B can be gained from a description of our stimulus in the Fourier domain. The power spectrum of $M(t)$ is flat up to frequencies of about $f$, where it cuts off due to the finite duration $b$ of the individual bits. Moreover, the area beneath this power spectrum is independent of $f$. Assuming that $f$ is large in comparison with the flicker fusion frequency of the human visual system, this means that the power of the internal signal (i.e., after passage through TF) is proportional to $1 / f$. Hence, the internal RMS contrast is proportional to $1 / \sqrt{f}=\sqrt{b}$.

2. There is a maximum for $\tilde{c}$ at intermediate values of the bit duration $b$. For the choice of parameters of the pulse response function $h(t)$ used in Figure A2, the maximum occurs for $b=$ $20 \mathrm{msec}(f=50 \mathrm{~Hz})$ and equals $\tilde{c}=2.4$. The fact that internal contrast $\tilde{c}$ is higher than $1(100 \%)$ is related to the bipolar nature of $h$ in Equation A8 that suppresses the average (timeindependent) part of the signal $M(t)$ (the denominator in Equation A3) relative to the fluctuations in $M(t)$. 
3. For very high values of $b$, the internal contrast reverts back to 1 (not shown in Figure A2). This corresponds to the situation that $b$ is much larger than the time scale $t_{0}$ of the pulse response function $h$. We never used this situation in our experiment.

The highest clock frequencies that we used in Experiment 1 were about $3000 \mathrm{~Hz}(b \approx 0.3 \mathrm{msec})$. From Figure $\mathrm{A} 2 \mathrm{~B}$, we can read off that $\tilde{c}^{2}$ is about 0.18 for this value of $b$. Thus, it follows that the (estimated) internal contrast was always larger than about $0.4(40 \%)$ for the stimuli employed in this experiment.

For the case that the bit duration $b$ is small in comparison with the time scale $t_{0}$ (the linear part in Figure A2B), we can simplify Equation A7 by approximating

$$
A(t)=\frac{M^{2} b}{4} \delta(t)
$$

Then,

$$
\begin{aligned}
<[\tilde{M}(t)-<\tilde{M}>]^{2}> & =\frac{M^{2} b}{4} \int h^{2}\left(\frac{t}{t_{0}}\right) d t \\
& =\frac{M^{2} b t_{0}}{4} \int h^{2}(y) d y .
\end{aligned}
$$

Substituting Equation A10 and Equation A4 into Equation A3, we find

$$
\tilde{c}=\left(\frac{b}{t_{0}}\right)^{1 / 2} \frac{\left[\int h^{2}(y) d y\right]^{1 / 2}}{\int h(y) d y} .
$$

Using this expression, we can translate the dependence that we observed in the main study: $T^{-}(:) n^{\beta_{-}}$(Equation 3 ) with $\beta_{-}=$ 0.47 from Table 2 into an estimate of the dependence of $T^{-}$on internal contrast $\tilde{c}$.

From Equation A11, we have $\tilde{c}(:)\left(b / t_{0}\right)^{1 / 2}$. From Equation 1, with $f=1 / b$, we derive $b=T / n$; thus $\tilde{c}(:)\left(T / n t_{0}\right)^{1 / 2}$. Rewriting this expression yields $n(:) \tilde{c}^{-2} T / t_{0}$, substantiating our claim in the main paper that $n$ is inversely related to (internal) contrast. Substituting this relationship between $n$ and $\tilde{c}$ into Equation 3 in the main paper yields

$$
T(:)\left(\frac{T}{t_{0} \tilde{c}^{2}}\right)^{\beta} \text {. }
$$

Solving Equation A12 for $T=T^{-}$by using the value $\beta^{-}=0.47$ from Table 2, we find

$$
T^{-}(:) t_{0}^{\frac{-\beta}{1-\beta_{-}}} c^{\frac{-2 \beta_{-}}{1-\beta_{-}}}=t_{0}^{-0.9} \tilde{c}^{-1.8} .
$$

Thus, we conclude that the seemingly innocuous exponent $\beta^{-} \approx$ 0.5 from Table 2 actually hides a strong dependence of $T^{-}$on the hypothetical internal contrast $\tilde{c}$ as defined in this appendix. In future experiments, it would be useful to determine whether there is a similar strong dependence of asynchronicity thresholds on the external contrast $c$ of the dynamic luminance variations. This would be interesting, since many experiments indicate a contrast saturation at stimulus contrasts above a few percent in human motion vision (see Stone, 1990, for a recent review).

\section{APPENDIX B \\ The Stochastic Error in the Threshold Delays}

A typical threshold determination would consist of one 30trial run that gave $27(90 \%)$ correct answers, and a second 30trial run with $21(70 \%)$ correct answers, where the delay values for both runs differed by a factor 2 .
From the binomial distribution that (we assume) governs the response behavior of the observer, it follows that the two values above have a standard deviation of 1.6 (for the case of $90 \%$ correct answers) respectively 2.5 (for the case of $70 \%$ correct answers); thus their mean has an expected error of $1 / 2\left[(1.6)^{2}+\right.$ $\left.(2.5)^{2}\right]^{1 / 2}=1.5$, which is $25 \%$ of their difference $(27-21)$. Hence, the location of the mean cannot be determined more accurately than $1 / 4$ of the delay interval between the two data points, which corresponds (on a logarithmic delay axis) with a standard deviation in the estimate for the threshold $(80 \%$ correct) delay of about $20 \%$.

\section{APPENDIX C \\ Consequences of the Inability to Perceive Anticorrelation}

In Experiment 2, we showed that human observers are unable to sense reversed motion from light source anticorrelation in situations where they do see (nonreversed) motion under light source correlation. Here we show that this result excludes any reasonable version of a motion-opponent Reichardt correlation model (or equivalently [Adelson \& Bergen, 1985; van Santen \& Sperling, 1985], in a motion energy model).

The class of opponent detectors excluded is basically the one shown in Figure $\mathrm{C} 1$. This class is very general; it includes as special cases motion-opponent versions of standard Reichardt models in which receptive field $\mathrm{RF}_{2}$ is a shifted copy of receptive field $R F_{1}$, and temporal filter $T F_{2}$ is a delayed copy of filter $\mathrm{TF}_{1}$, but also opponent versions of quadrature (motion energy) models in which $\mathrm{SF}_{2}$ is a Hilbert transformed copy of $\mathrm{SF}_{1}$ (and, likewise, $\mathrm{TF}_{2}$, the Hilbert transform of $\mathrm{TF}_{1}$ ).

The spatiotemporal luminance pattern that we used in Experiments 1 and 2 is of the form (see Equation 2):

$$
J \pm(x, t)=P(x) M(t) \pm P(x-s) M(t-T) .
$$

The " + " sign is for the case of full correlation (Experiment 1); the "-" sign, for the case of anticorrelation (Experiment 2). Note that we dropped mean luminance from $M(t)$, which does not lead to any loss of generality because the opponent detector of Figure $\mathrm{C} 1$ does not respond to a constant luminance. The spatial luminance function $P(x)$ was point-like in our presentation, but this turns out to be not at all crucial, because any function $P(x)$ would serve in the derivation presented below. Likewise, the fact that $M(t)$ was essentially white noise in our experiment is not crucial at all; any function $M(t)$ would serve in the derivation below. In fact, we give the proof below for a still more general dynamic luminance pattern:

$$
I \pm(x, t)=P(x) M(t) \pm Q(x) N(t) .
$$

If the detector in Figure $\mathrm{Cl}$ responds to the sum of two arbitrary spatiotemporal separable dynamic luminance patterns, it reverses its response if one presents the difference of these dynamic luminance patterns.

The proof of this detector output reversal is very simple. We sketch it here and leave the details to the reader. We denote the output of the (serial) combination of the spatial filter $\mathrm{SF}_{i}(i=1,2)$ and the temporal filter $\operatorname{TF}_{j}(j=1,2)$ to the input of Equation $\mathrm{C} 2$ as

$$
\mathrm{SF}_{i} \mathrm{TF}_{j}=P_{i}(X) M_{j}(t) \pm Q_{i}(X) N_{j}(t),
$$

where we introduced the position tag $X$ of the detector to stress its arbitrary location relative to the stimulus. Then the detector output of Figure $\mathrm{Cl}$ can be denoted symbolically as 
$\left[\mathrm{SF}_{1} \cdot \mathrm{TF}_{1}\right] \times\left[\mathrm{SF}_{2} \cdot \mathrm{TF}_{2}\right]-\left[\mathrm{SF}_{1} \cdot \mathrm{TF}_{2}\right] \times\left[\mathrm{SF}_{2} \cdot \mathrm{TF}_{1}\right]$

One can easily show that the quadratic terms $P_{1} P_{2} M_{1} M_{2}$, etc. that arise from the multiplications (" $X$ ") in Equation C4 cancel in the final difference (" - ") operation; hence, only mixed terms such as $P_{1} Q_{2} M_{1} N_{2}$, etc., survive. However, these mixed terms always carry the " \pm "' sign from Equation C2. Hence, it follows that the output of the detector in Figure $\mathrm{C} 1$ undergoes a complete reversal under the sign manipulation " \pm " in Equation C2. Moreover, one can easily show that exactly the same output sign reversal occurs if one presents the detector

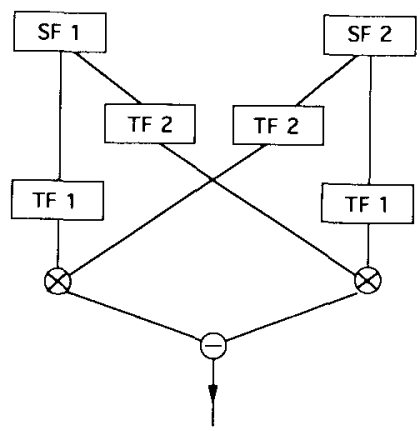

Figure C1. The basic structure of a detector of the motionopponent type. not with the input pattern $I_{+}(x, t)$ from Equation $\mathrm{C} 2$, but with the input pattern

$$
I_{+}^{R}(x, t)=P(x) N(t)+Q(x) M(t)
$$

that represents the physically opposite motion direction. Thus, the detector responses under input patterns $I_{-}(x, t)$ from Equation $\mathrm{C} 2$ and $I_{+}^{R}(x, t)$ from Equation $\mathrm{C} 5$ are equal. This is true already without temporal filtering of the output of the single, arbitrarily placed detector. Thus, no combination of the outputs of different detectors, nor any temporal filtering of detector output, can possibly destroy the equivalence.

Hence we have a very strong result. For symmetry reasons, one expects that if a certain input pattern $I_{+}(x, t)$ from Equation $\mathrm{C} 2$ gives rise to a motion percept, the input pattern $I_{+}^{R}(x, t)$ from Equation C5 gives rise to the same motion percept--except that the perceived motion direction is reversed. Because of the equality of detector responses under input patterns $I_{+}^{R}(x . t)$ and $I_{-}(x, t)$ in a motion-opponent system, the stimulus $I_{-}(x, t)$ should then give rise to the same reversed motion percept. In Experiment 2, we have an input pattern of the form $I_{-}(x, t)$ that does not give rise to a motion percept though the corresponding pattern $I_{+}(x, t)$ from Experiment 1 does give rise to a motion percept. This pattern combination $I \pm(x, t)$ is thus a counterexample (possibly coming from a large class) that refutes the motion-opponency scheme of Figure $\mathrm{Cl}$ as a model for human front-end motion vision.

(Manuscript received November 24, 1991; revision accepted for publication June 17, 1993.) 